\title{
Limited evidence suggests that mouthrinses may be effective in reducing oral malodour
}

\author{
Abstracted from \\ Blom T, Slot D, Quirynen M, Van der Weijden G. \\ The effect of mouthrinses on oral malodor: a systematic review. \\ Int J Dent Hyg 2012 Mar 19. doi: 10.1111/j.1601-5037.2012.00546.x. [Epub ahead of print] \\ Address for correspondence: DE Slot, Academic Center for Dentistry Amsterdam (ACTA) \\ University of Amsterdam and VU University Amsterdam, Department of Periodontology, \\ Gustav Mahlerlaan 30041081 LA Amsterdam The Netherlands. E-mail: d.slot@acta.nl
}

\section{Question: Are mouthrinses effective in reducing oral malodour?}

Data sources Medline, The Cochrane Central Register of Controlled Trials (CENTRAL) and Embase databases were searched.

Study selection Screening and selection of studies was conducted independently by two authors, with randomised controlled trials (RCTs) or controlled clinical trials (CCTs) conducted in adults 18 years or older being included. Studies used the outcome measures of volatile sulphur compounds (VSCs), organoleptic measurement (OM) and tongue coating (TC). Studies with a duration of greater than one day were included. Selection was restricted to English language papers.

Data extraction and synthesis Study quality was assessed by two authors with studies being separated into short- term ( $<3$ weeks) and longer-term study period groups ( $\geq 3$ weeks). Meta-analysis was not conducted because of heterogeneity in the study designs, products used, outcome measures and data presentation.

Results Twelve RCTs were included (six cross-over studies and six parallel design). The short-term studies ranged from four days to two weeks and the long-term studies from three to four weeks. The risk of bias was assessed as high for two studies, moderate for three and low for seven. Nearly all mouthwashes with active ingredients had beneficial effects in reducing oral malodour in both short- and longer-term studies.

Conclusions The most compelling evidence was provided for chlorhexidine mouthwashes, and those that contained a combination of cetyl pyridinum chloride and zinc provided the best evidence profile on oral malodour. Little data with respect to tongue coating were available, and none of the studies showed a beneficial effect for this parameter.

\section{Commentary}

The prevalence of oral malodour (halitosis) has been reported to be as high as $50 \%$ although the reliability of the data has been questioned. The majority of cases are now considered to have an oral cause with less than $10 \%$ having a non-oral cause. The major component of oral malodour $(\mathrm{OM})$ are the volatile sulphur compounds (VSCs) and treatment appears to hinge on their reduction. This current review focuses on the use of mouthrinses to treat OM. A previous Cochrane review tackling the same question was published in $2008^{1}$ so this review could be viewed as an update as the same main databases were searched. One major difference between this review and the Cochrane review highlighted by the review authors is treatment times considered. The Cochrane review only considered treatment times of one week or greater whereas this current review considered treatment times of one day or more. This decision allowed the authors to include more trials, (12), compared with the Cochrane review, (five), as despite the passage of time only two studies included here were completed after the publication of the Cochrane review.

While the authors reported the assessment of risk of as low for seven of the included studies in the discussion, they indicate that if they had included allocation concealment (a key component of bias protection) only one of the studies would be considered to have a low risk of bias. While I agree that this may be a reporting issue rather than a study conduct it does form an important element of assessing potential bias.

Although the results of the review suggest beneficial effect from almost all of the mouthwashes with active ingredients in both the short- and long-term, the qualities of the studies mean that the evidence is not yet reliable enough to make informed decisions.

The authors also include a useful discussion of the main methods of assessing OM citing the recent paper assessing the relationship between organoleptic scores and the Halimeter ${ }^{\circledR}$ or gas chromatography, indicating that correlations between the three methods of breath measurement were high. However, it is clear from this and the previous Cochrane review that more high quality trials are needed to provide definitive answers to this problem. With this in mind it would be helpful to agree which standard assessment of OM should be used in studies in order to allow future systematic reviewers to combine trials from different research group more easily.

\section{Practice points}

- There is limited evidence that mouth rinses with active ingredients (chlorhexidine and cetyl pyridinum chloride with zinc) may be effective in reducing oral malodour.

Derek Richards Centre for Evidence-based Dentistry, Oxford, UK

1. Fedorowicz Z, Aljufairi H, Nasser M, Outhouse TL, Pedrazzi V. Mouthrinses for the treatment of halitosis. Cochrane Database Syst Rev 2008; Issue 4. Art. No: CD006701.

2. Saad S, Greenman J, Shaw H. Comparative effects of various commercially available mouthrinse formulations on oral malodor. Oral Dis 2011; 17: 180-186.

Evidence-Based Dentistry (2012) 13, 121. doi:10.1038/sj.ebd.6400902 